Cell Research (2002); 12(5-6):321-329

http://www.cell-research.com

REVIEW

\title{
Stem cell pluripotency and transcription factor Oct4
}

\author{
GuAng Jin PAN ${ }^{1}$, Zeng YI CHANG ${ }^{1}$, Hans R. SCH? ER ${ }^{2}$, DuANQING PEI ${ }^{1,3} *$ \\ ${ }^{1}$ Departments of Pharmacology and Biological Sciences \& Biotechnology, Schools of Sciences and Medicine, \\ Tsinghua University, Beijing 100084, China \\ ${ }^{2}$ Center for Animal Transgenesis and Germ Cell Research, School of Veterinary Medicine, Department of Animal \\ Biology, University of Pennsylvania, New Bolton Center, 382 West Street Road, Kennett Square, PA \\ 19348, USA, \\ ${ }^{3}$ Department of Pharmacology, University of Minnesota, Minneapolis, MN 55455, USA
}

\begin{abstract}
Mammalian cell totipotency is a subject that has fascinated scientists for generations. A long lasting question whether some of the somatic cells retains totipotency was answered by the cloning of Dolly at the end of the 20th century. The dawn of the $21^{\text {st }}$ has brought forward great expectations in harnessing the power of totipotentcy in medicine. Through stem cell biology, it is possible to generate any parts of the human body by stem cell engineering. Considerable resources will be devoted to harness the untapped potentials of stem cells in the foreseeable future which may transform medicine as we know today. At the molecular level, totipotency has been linked to a singular transcription factor and its expression appears to define whether a cell should be totipotent. Named Oct4, it can activate or repress the expression of various genes. Curiously, very little is known about Oct4 beyond its ability to regulate gene expression. The mechanism by which Oct4 specifies totipotency remains entirely unresolved. In this review, we summarize the structure and function of Oct4 and address issues related to Oct4 function in maintaining totipotency or pluripotency of embryonic stem cells.
\end{abstract}

Key words: Oct4, ES cells, pluripotency, totipotency, stem cells, cloning.

\section{INTRODUCTION}

Totipotency, a term most likely used for the first time in 1909, refers to the capacity of a portion of an organism to generate or regenerate an entire new organism[1]. In higher mammals, totipotency has been proven difficult to achieve. Instead, multipotency and pluripotency of mammalian cells have been explored in recent decades. By injecting embryonic carcinoma (EC) cells derived from the central portions of mouse embryoid bodies into blastocysts, Mintz and colleagues demonstrated that

* Corresponding author: Dr. Duanqing PEI

Fax: 001-612-624-3952; E-mail: Duanqing@aol.com
EC cells can contribute to the development of most of the tissues and cell lineages in the newly formed mosaics[2],[3]. These observations led them to conclude that these EC cells remain multipotent, despite being passaged in vivo as an ascites tumor for 8 years[2], [3]. Culture conditions were subsequently established that allowed the isolation of pluripotent embryonic stem (ES) cells from mouse embryos[4]. The availability of mouse ES cells led to the development of gene targeting technology, which is widely used today[5]. Given the capacity of ES cells to generate virtually any cell type in the body, intense efforts have focused on harnessing the potential of human ES cells for medical applications. 
One important question to address is: How are ES cells derived? In human, the fertilization of an egg by a sperm generates a zygote that thirty hours later begins to divide. By the third to fourth day the embryo develops to a compact ball of twelve or more cells called a morula. After several more divisions, the morula cells begin to specialize and form a hollow sphere of cells called a blastocyst. The outer layer of the blastocyst is named the trophectoderm (TE) and the cells inside inner cell mass (ICM). The cells of the ICM are pluripotent stem cells that can give rise to all cell types of the three embryonic germ layers, i.e., ectoderm, mesoderm, and endoderm, and the germ cell lineage. In recent years, ES cell lines have been derived from the ICM of human embryos [6]. The established human ES cell lines had the ability to renew themselves continuously under appropriate culture conditions and to develop into cell lineages from all three embryonic germ layers[6]. Thus, human ES cells may become an unlimited source of cells or tissues for transplantation therapies involving organs or tissues such as liver, nervous system, pancreas and blood. Despite a tremen- dous interest in ES cells, relatively little is known about what defines their pluripotency and what drives ES cells to differentiate into specific cell types.

The development of the mammalian embryo is controlled by regulatory genes, some of which regulate the transcription of other genes[7],[8]. These regulators activate or repress patterns of gene expression that mediate phenotypic changes during stem cell differentiation. Oct4 (also known as Oct3) belongs to the POU (Pit-Oct-Unc) transcription factor family[8]. The POU family of transcription factors can activate the expression of their target genes through binding an octameric sequence motif of an AGTCAAAT consensus sequence[7],[9]. Recent evidence indicates that Oct4 is almost exclusively expressed in ES cells (Fig 1)[10-13]. During embryonic development, Oct4 is expressed initially in all blastomeres. Subsequently, its expression becomes restricted to the ICM and downregulated in the $\mathrm{TE}$ and the primitive endoderm. At maturity, Oct4 expression becomes confined exclusively to the developing germ cells[9],[11]. Targeted disruption of Oct4 in mice has produced embryos devoid of a

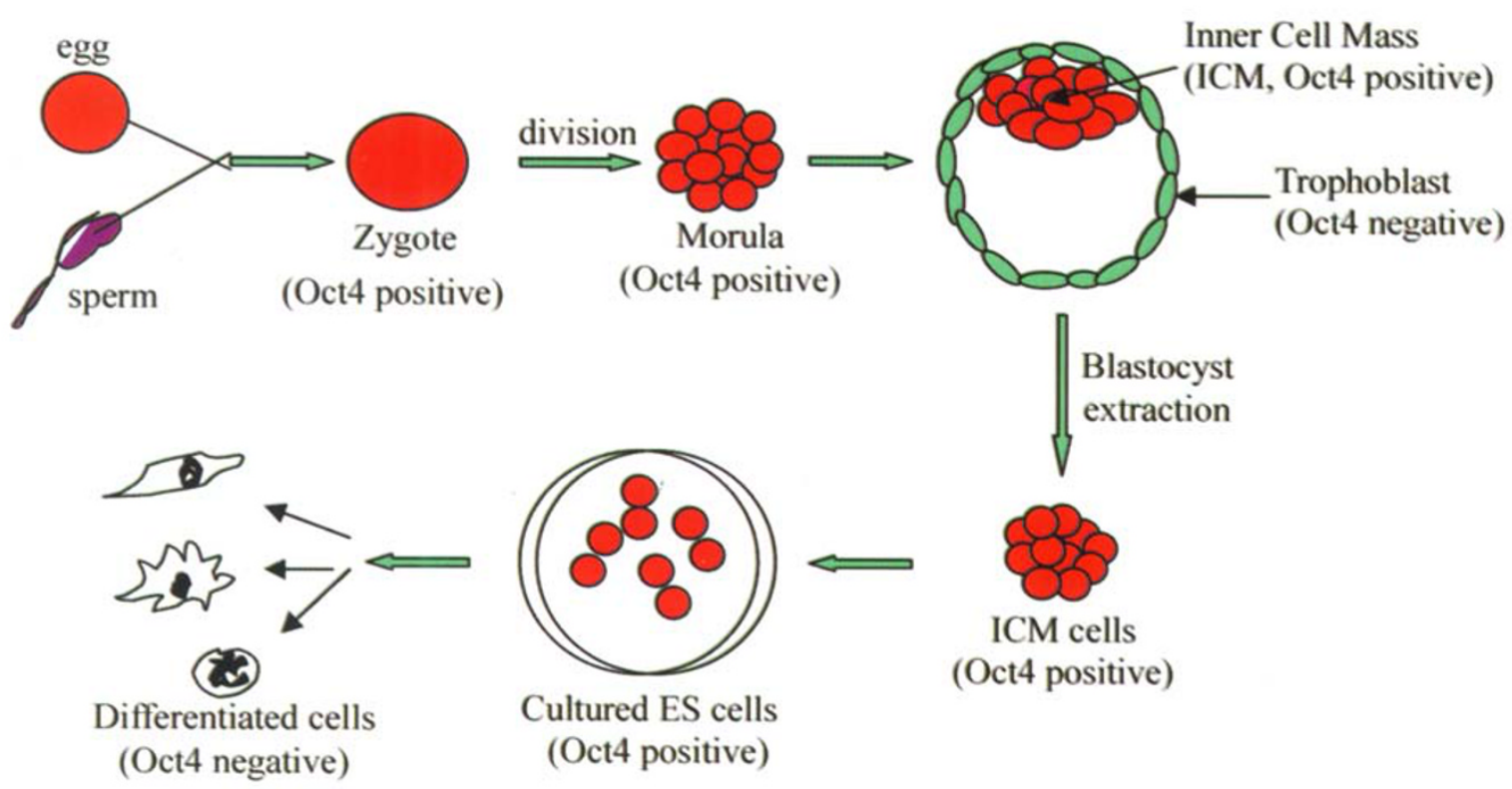

Fig 1. ES cells and Oct4 expression The isolation and differentiation of ES cells in vitro are illustrated schematically starting with the fertilization of an egg by a sperm to form a zygote. At the blastocyst stage, inner cell mass (ICM) becomes visible and can be extracted and cultured in vitro to form embryonic stem (ES) cells. Cultured ES cells can be induced to differentiate into various cell types that are negative for Oct4. The stages of Oct4 expression are noted and the cells with Oct4 expression are marked in red colour. There is a general correlation between Oct4 expression and totipotency[13], [14]. 
pluripotent ICM[12], suggesting that Oct4 is required for maintaining pluripotency. Furthermore, quantitative analysis of Oct4 expression revealed that a high level of Oct4 expression drives ES cells towards the extra-embryonic mesoderm or endoderm lineages, while those with a low level of Oct4 become trophectodermal cells; ES cells with a normal level of Oct4 remain pluripotent[13],[14]. Thus, it has been proposed that Oct4 is a key regulator of stem cell pluripotency and differentiation[9],[11]. Further

A

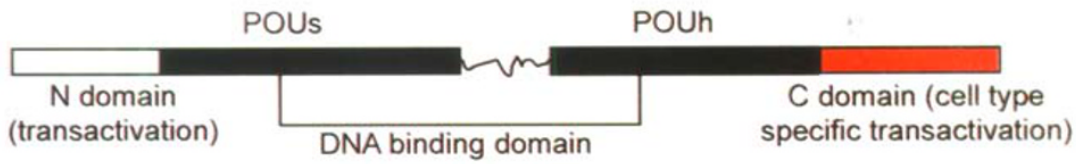

B

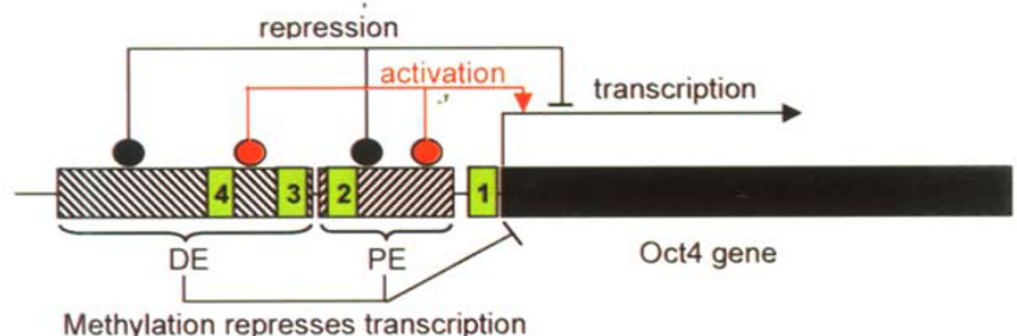

Methylation represses transcription

C

1)
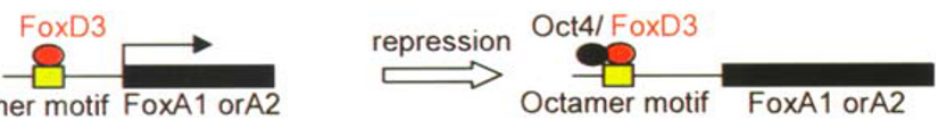

2)
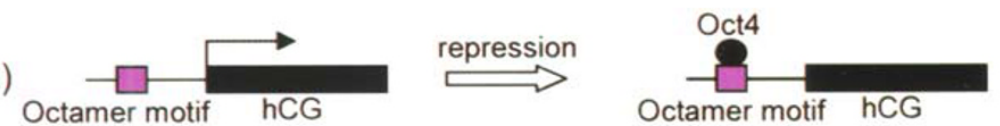

3)
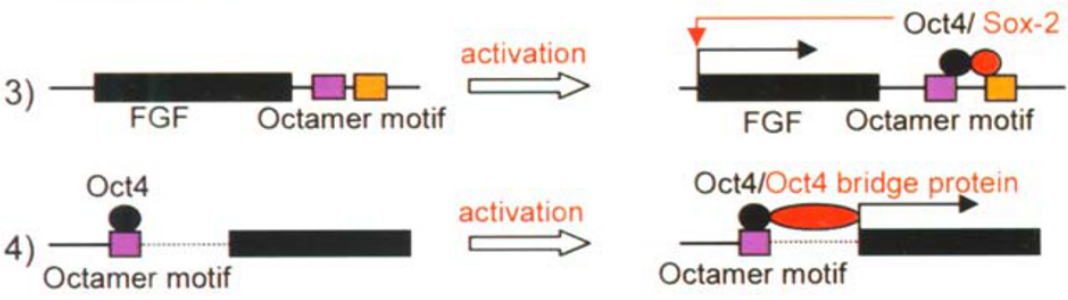

5)
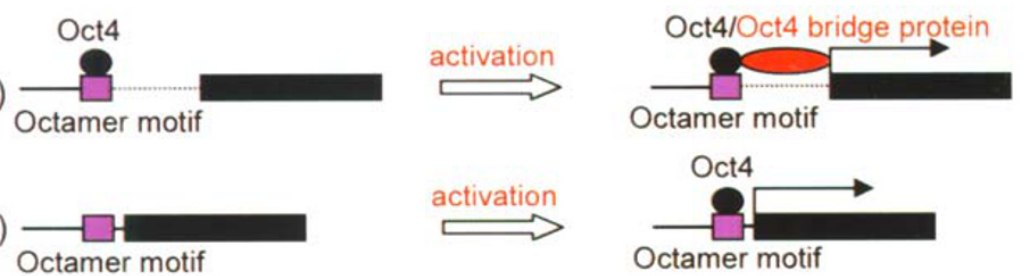

Fig 2. Structure and Function of Oct4 A. a schematic illustration of Oct4 domains. Note the C domain behaves differently from the $\mathrm{N}$ domain with respect to cell type-specific transactivation. B. The upstream regulatory elements of the Oct4 gene. DE, distal enhancer, and PE, proximal enahncer, are important for regulating Oct4 expression. There are 4 regions that are highly conserved among human, bovine and mouse Oct4 promoter/enhancer elements, shown as green box 1 through 4 relative to DE and PE. Conserved region 1 (CR1) is downstream of PE and immediately upstream of exon 1 . Each enhancer contains multiple potential binding sites for transcription factors that can either activate (red) or repress Oct4 expression. In addition, methylation in these regions represses Oct4 expression in differentiated cells. C.Modes of action of Oct4 on different target genes. Oct4 represses gene expression either indirectly by neutralizing activators such as FOXD3 (example 1), or directly by binding to promoters (example 2). Oct4 also acts as an activator of gene transcription by binding to octamer sites located upstream (example 4 and 5) or downstream (example 3) of target genes. In the simplest mode, Oct4 binds to octamer sites immediately upstream of the promoter to activate gene expression directly (example 5). Alternatively, Oct4 can synergize with other factors like Sox2 to activate gene transcription (example 3). When located at a considerable distance, as in example 4, adaptor proteins must be involved to bridge Oct4 to the basic transcription machinery for transcriptional activation. 
investigation of Oct4 may help unravel the molecular and cellular mechanisms of stem cell pluripotency. In this review, we focus on Oct4 and discuss its structure and function in the context of stem cell renewal and differentiation.

\section{Structure and function of Oct4}

The hallmark feature of the POU family of transcription factors is the POU domain, which consists of two structurally independent subdomains: a 75 amino acid amino-terminal POU specific (POUs) region and a 60 amino-acid carboxyl-terminal homeodomain (POUh) [see Fig 2A][7]. Both domains make specific contact with DNA through a helix-turnhelix structure and are connected by a variable linker of 15 to 56 amino-acids[15]. Regions outside the POU domain are not critical for DNA binding and exhibit little sequence conservation. The $\mathrm{N}$-terminal domain ( $\mathrm{N}$ domain) is rich in proline and acidic residues, while the $\mathrm{C}$-terminal domain ( $\mathrm{C}$ domain) is rich in proline, serine and threonine residues[7]. The $\mathrm{N}$ domain has traditionally been accepted for its role in transactivation[16]. More recent data suggest that the $\mathrm{C}$ domain also plays a role in transactivation [17]. Brehm et al replaced the POU DNA binding domain with those from other transcription factors, for example, the heterologous yeast Gal4 DNA binding domain[16]. This replacement did not affect its transactivation function, suggesting that general transactivation function can be transferred to unrelated DNA binding domains. It was subsequently demonstrated that the activity of Oct4 $\mathrm{C}$ domain is cell type specific and is regulated through phosphorylation, whereas the $\mathrm{N}$ domain is not[16], [18],[19]. The cell type specificity was observed only if the $\mathrm{C}$ domain was linked to the POU domains of Oct4 and Oct- 2, but not to Pit-1 or the Gal4 DNA binding domain[16]. This finding suggests that Oct4 POU-domain may function differently by serving as interaction sites for cell type-specific regulatory factors.

Since the cell-type-specific activity of regulatory factors ensures the expression of target genes in an orderly fashion during development, Oct4 and its functional partners may be regulated in a specific manner throughout mammalian embryogenesis. Indeed, Oct 4 is expressed by germ cells from the totipotent zygote to the highly specialized oocyte [9], [20],[21]. It is likely that Oct4 may function in con- cert with other regulators to activate specific target genes in specific cell types at defined developmental stages. The fact that the $\mathrm{N}$ domain differs from the $\mathrm{C}$ domain in activity and cell type specificity may help explain the functional diversity for Oct4. Furthermore, the $\mathrm{C}$ domain may activate certain targets, which do not respond to the $\mathrm{N}$ domain during development[16]. Examination of Oct4 structure with respect to its diverse biological function is only the starting point in unraveling the regulatory circuits responsible for maintaining stem cell pluripotency and controlling the differentiation of stem cells to various cell types. Certainly, more detailed work will be required to understand the structural basis of Oct4 functionality.

\section{Regulation of Oct4 expression}

Given its critical role in maintaining pluripotency, Oct4 activity must be tightly regulated to ensure the continuity of the germline and proper differentiation of various tissues and organs. In the mouse, Oct4 mRNA is present in mature oocytes[20]. At the eightcell stage, Oct4 expression reaches a much higher level[21]. Subsequently, Oct4 expression becomes restricted to the ICM, and is downregulated in the TE and primitive endoderm (see Fig 1)[21]. Later on in embryo development, Oct4 expression is only maintained in primordial germ cells (PGCs) [9, 20]. Hansis et al examined Oct4 expression in human blastocysts. The ICM and TE of 17 human blastocysts were separated and Oct4 mRNA level was individually assessed by RT-PCR[22], [23]. The results demonstrated that the mean Oct4 expression was 30 times higher in totipotent ICM cells than in differentiated TE cells (Fig 1)[22],[23]. These studies suggest that the expression pattern of Oct4 is very similar between mouse and human cells.

Expression of Oct4 is regulated at the transcription level by cis-acting elements located upstream of the Oct4 gene and methylation of chromatin structure (Fig 2B)[24]. By analyzing the expression of the LacZ reporter gene under the control of a $18 \mathrm{~Kb}$ fragment from Oct4 genomic locus, Yeom et al identified two elements, which they named proximal enhancer $(\mathrm{PE})$ and distal enhancer (DE) that may regulate the cell -type-specific expression of Oct4 (Fig 2B)[25]. By using in vivo footprinting, they identified the precise binding sites for transcription factors within these two enhancers[25]. One site, 
named $1 \mathrm{~A}$, was identified within the $\mathrm{PE}$, and another site, named 2A, within the DE. Both sites exhibit nearly identical sequence homologous to the GC box and are crucial for the activity of PE and $\mathrm{DE}$, respectively[25]. But there was no further evidence to demonstrate the involvement of these two enhancers in stem cell specific activities in vivo. On the other hand, Nordhoff et al comparatively analyzed the human, bovine, and murine Oct4 upstream promoter sequences and revealed four conserved regions of homology (CR1 to CR4) between these species (66-94\% conservation)[26]. They found that element $1 \mathrm{~A}$ in $\mathrm{PE}$ (see above) is located approximately half way between CR2 and CR3[26]. A putative Sp1/Sp3 binding site and an overlapping hormone responsive element (HRE) in CR1 were found to be identical in all three species[26]. In addition, there were a large number of $\mathrm{CCC}(\mathrm{A} / \mathrm{T}) \mathrm{CCC}$ motifs, which exhibit various levels of homology within the upstream regions[26]. These sequences may be essential for Oct4 expression, thus further experimental investigation is necessary. In addition, Hummelke and Cooney reported that germ cell nuclear factor (GCNF), an orphan nuclear receptor, could repress Oct4 gene activity by specially binding to the sequence within the $\mathrm{PE}[27],[28]$. In agreement to this, GCNF expression inversely correlates with Oct4 expression in differentiating embryonic cells[27], [28]. In mouse embryos deficient in GCNF, the expression of Oct4 is no longer limited within the germ cell linage after gastrulation[28], suggesting that GCNF is responsible for the repression of Oct4 gene expression during stem cell differentiation. Further dissection of the Oct4 gene promoter/enhancers will reveal the precise cis-acting elements that bind to corresponding trans-acting factors, which act in concert to govern the lineage-specific expression or repression of Oct4.

In addition to the cis-acting elements, there are additional mechanisms that may regulate the activity of Oct4. One hypothesis is that the steady state level of Oct 4 in totipotent cells may be a consequence of the establishment of active chromatin rather than the function of transcription activators. Ben-Shushan et al reported that the extinction of Oct4 activity in stem cells-fibroblast hybrid cells was accompanied by rapid methylation of regulatory sequences such as $\mathrm{PE}$ and $\mathrm{DE}$ in the Oct4 promoter/enhancer re- gion[24]. Jaenisch suggested that there must be a wave of de novo methylation occurring in the somatic cells of the embryo[29]. These studies are consistent with the idea that methylation of the Oct4 regulatory sequences such as $\mathrm{PE}$ and $\mathrm{DE}$ shuts down Oct4 expression. PGCs, arising from extra-embryonic mesoderm and maintaining a steady level of Oct4 expression, may have a mechanism to prevent the methylation of their genome, at least within the Oct4 regulatory sequence[30]. On the other hand, the maintenance of Oct4 expression in PGCs and oocytes may be due to the escape of chromatin from general reprogramming by methylation that occurs in epiblast cells at the time of gastrulation[31]. It remains to be determined how ES cells integrate regulation by transcription factors and DNA methylation to either retain pluripotency or undergo differentiation into various cell types.

\section{The function of Oct4 in development and pluripotency}

In an effort to define the relationship between Oct4 expression and stem cell pluripotency, Niwa et al measured the levels of Oct4 expression at various ES cell states[13],[14]. These results indicated that Oct 4 controls the pluripotency of stem cells in a quantitative fashion. Specifically, they determined that high level of Oct4 expression drives ES cells to endoderm and mesoderm lineages, while stem cells with low level of Oct4 differentiate into TE[13],[14]. Only a "normal level" of Oct4 can retain stem cells in a pluripotent state[13],[14]. These observations suggest that Oct4 is different from many known transcription regulators that appear to function in a binary on-off manner. In some cases, Oct4 can act as a repressor of target genes whereas in other cases, it acts as an activator (Fig 2C). For example, Oct4 motifs were reported within the proximal promoters of $a$ and $b$ subunit of human chorionic gonadotropin (hCG) genes[32], [33]. Oct4 serves as a repressor of both of these genes through binding to the octamer motifs in stem cells[32], [33]. In differentiated TE, Oct4 is downregulated and no longer able to trans-repress hCG expression, signaling the reversal of a newly established gene expression pattern in these cells.

Oct4 may function through other transcription factors to activate or repress target genes. Members 
of the Forkhead Box (Fox) family have a winged-helix DNA binding structure and are strongly implicated in early embryonic lineage decisions, especially in the development of the endoderm and subsequent endodermal organogenesis[34]. $\mathrm{FoxD}_{3}$, a member of this family, could bind to and activate the promoters of other members of this family, e.g., FoxA1 and FoxA2, while FoxA1 and FoxA2 are critical for the embryonic development of endodermal foregut organs[35]. Guo et al reported that Oct4 could repress the expression of FoxA1 and FoxA2 through an interaction with the DNA binding domain of their activator $\mathrm{FoxD}_{3}[35]$. Since Oct4 does not bind to the promoters of FoxA1 and FoxA2, it behaves as a corepressor of these promoters. This report suggests that Oct4 could prevent the differentiation of ES cell lineages by acting like a corepressor of lineage-specific transcription factors like $\mathrm{FoxD}_{3}$ [35]. Silencing of tau interferon genes (IFN $\tau$ ) appears to be mediated by Oct4. IFN $\tau$ is expressed exclusively in the TE of bovine embryos and activated through the Ets- 2 binding enhancer[36]. Ezashi et al reported that Oct4 and Ets-2 could form a complex through interaction between the Oct4 POU domain and the DNA binding domain of Ets-2, and as a result quench the transactivation function of Ets-2[37]. In trophectodermal cells, Oct4 is downregulated, thus, alleviating the co-repression of Ets-2 to allow the TE specific genes such as IFN $\tau$ to be expressed[37]. These findings provided evidence that the developmental switch could be accomplished by the loss of Oct4 mediated silencing of key genes.

A direct mechanism by which Oct 4 can exert regulatory function involves transactivation of target genes in stem cells (Fig 2C). Oct4 can transactivate its targets either proximally or remotely, depending on the location of its binding sites on the target promoters. Acting over a long distance, Oct4 may enlist the assistance of stem cell-specific coactivators that can bridge a remotely bound Oct4 protein to the basal transcription machinery (Fig 2C). Quite unexpectedly, the adenovirus (Ad) E1A oncoprotein was found to be able to mimic the function of such stem cell-specific coactivators[38],[39]. Another oncoprotein, HPV-E7, was also reported to have a similar role in Oct4-mediated gene activation[19]. Both proteins function as the bridging factors connecting a remotely bound Oct4 molecule to the gen- eral transcription machinery. Oct4 can also synergize with other transcriptional factors bound to the nearby cis-acting elements of target promoters. One such example concerns the regulation of FGF4 expression. FGF4 is a stem cell-specific growth factor, and has an enhancer element located within the 3 '-untranslated region (UTR) of the gene, which is responsible for its stem cell-specific expression [40]. This enhancer contains an octamer motif adjacent to a binding site to which Oct4 and the high mobility group (HMG) transcription factor Sox-2 bind cooperatively to activate transcription synergistically[41]. This synergism is most likely mediated by protein-protein interactions[42]. In the absence of Sox-2, Oct4 is not sufficient for FGF4 enhancer activity, even in the presence of the bridging factor E1A[42]. In addition, the formation of Oct4/ Sox-2 complex also appears to be a reciprocal event, since the complex could unmask latent activation domains in both proteins, thus leading to transcriptional activation[43]. Interestingly, like the regulatory elements in FGF4 enhancer, the Sox-2 enhancer also has an octamer motif that can be regulated by Oct4/Sox-2 synergistically[44]. The stem cell-specific gene Utf1 is regulated through synergistic action of Oct4/Sox-2[45]. These observations illustrate the versatility of Oct4, acting either as a suppressor of genes responsible for ES cell differentiation or as a transactivator of genes known to retain the pluripotency of ES cells. As such, Oct4 can be considered as the primary factor that determines the fate of ES cells between self-renewal and differentiation.

\section{Oct4 and somatic cloning}

In higher mammals, somatic clones generated by nuclear transfer often fail to develop at early embryonic stages[46]. The developmental rates of mammalian species that have been successfully cloned so far, have been extremely low, for example, in mouse this is at 3\%[46]. A vast majority of somatic cell clones could be transferred at the morula and blastocyst stage, but fail to develop past 6-7 days post coitum (dpc), with implantation rates less than $10 \%$ of the total number of transferred embryos[47],[48]. These data suggest that most clones are not able to develop past the preimplantation or early postimplantation stages. A favored hypothesis in this regard is that 
the embryonic cells created by somatic nuclear transfer are not able to reprogram the transferred nucleus to a state equivalent to that of an early embryonic nucleus from a zygote[47], [48]. As discussed further above, Oct4 controls the expression of several genes during early development, including FGF4, IFN $\tau$, Sox-2, hCG, Utf-1 and was also reported to regulate other downstream genes like Creatine kinase $\mathrm{B}[49]$. In order to determine the role of Oct4 in the development of cloned embryos, Boiani et al used Oct4 and the Oct4-GFP transgene as a marker to follow the reprogramming of cell pluripotency in clones, from the differentiated state of the nuclear donor cell to the pluripotent state of the ICM cells [50]. One surprising finding is that although the majority of clones failed to develop, temporal expressions of Oct4-GFP were observed in the majority of surviving cumulus clones undergoing development [50]. When the pattern of Oct4 expression was investigated in cloned blastocysts, only $34 \%$ of cloned embryos expressed Oct4 exclusively in ICM cells whereas the majority of control embryos did[50]. Over half (54\%) of them expressed Oct4 in both ICM and TE. These results demonstrate that although the reprogramming of Oct4 occurred rapidly in cloned embryos, its expression pattern was abnormal both temporally and spatially. This aberrant expression of Oct4 in cloned embryos may be associated with the abnormal expression of other crucial genes, leading to abnormalities at various embryonic stages. Therefore, the failure of cloned embryos to reprogram Oct4 expression could be the leading cause of low success rate in somatic cloning. Reprogramming of the transferred nucleus through transcription factors like Oct4, may thus hold the key to successful somatic cloning in higher mammals.

\section{Oct4 and Cancer}

Phenotypically, human preimplantation embryonic cells resemble cancer cells in many ways, especially in their ability to grow indefinitely. Both types of cells undergo deprogramming to a proliferating state and become immortal, self-renewing, and invasive. These similarities suggest that some embryonic genes may be re-expressed or re-activated in cancer cells. Palumbo et al reported that human testicular germ cell tumors [TGCTs] express a $1.5 \mathrm{~kb}$ alternative transcript of the platelet-derived growth factor (PDGF) alpha receptor gene[51]. Others have reported that Oct4 and three other novel embryonic genes are expressed in human tumors but not in normal somatic tissues[[52], in agreement with the hypothesis that embryonic genes are re-activated in tumor cells. Consistently, Jin et al discovered that the human breast cancer cell line, MCF7, expresses at least four POU gene products including Oct4[53]. Taken together, these studies suggest a link between Oct4 and tumorigenesis. Like ES cells, tumor cells exhibit a unique pattern of gene expression, and thus, may be under the control of one or more master regulators like Oct4. Therefore, an understanding of Oct4 function in stem cell biology could also lead to novel treatments for certain malignancies.

\section{ACKNOWLEDGEMENT}

This work is supported in part by the Tsinghua BaiRen Program (985). The authors wish to thank Dr Nanming Zhao of Tsinghua for much encouragement and support.

\section{REFERENCES}

[1] Mish FC. Webster' s ninth new collegiate dictionary 1988: pp1246.

[2] Mintz B, K Illmensee. Normal genetically mosaic mice produced from malignant teratocarcinoma cells. Proc Natl Acad Sci USA 1975; 72:3585-9.

[3] Illmensee K, B Mintz. Totipotency and normal differentiation of single teratocarcinoma cells cloned by injection into blastocysts. Proc Natl Acad Sci USA 1976; 73:54953.

[4] Evans MJ, MH Kaufman. Establishment in culture of pluripotential cells from mouse embryos. Nature 1981; 292:154-6.

[5] Capecchi MR. Altering the genome by homologous recombination. Science 1989; 244:1288-92.

[6] Thomson .A, J Itskovitz-Eldor, SS Shapiro, MA Waknitz, JJ Swiergiel, VS Marshall, JM Jones. Embryonic stem cell lines derived from human blastocysts. Science 1998; 282:1145-7.

[7] Scholer H. Octamania: the POU factors in murine development. Trends Genet. 1991; 7:323-9.

[8] Scholer H, S Ruppert, N Suzuki, K Chowdhury, P Gruss. New type of POU domain in germ line-specific protein Oct4. Nature 1990; 344:435-9.

[9] Pesce M, and H Scholer. Oct4: gatekeeper in the beginnings of mammalian development. Stem Cells 2001; 19: 271-8.

[10] Scholer H, G Dressler, R Balling, H Rohdewohld, P Gruss. Oct4: a germline-specific transcription factor mapping to the mouse t-complex. EMBO J 1990; 9:2185-95.

[11] Pesce M, H Scholer. Oct4: control of totipotency and 
germline determination. Mol Rep Dev 2000; 55:452-7.

[12] Nichols J, B Zevnik, K Anastassiadis, H Niwa, D KleweNebenius, I Chambers, H Scholer, A Smith. Formation of pluripotent stem cells in the mammalian embryo depends on the POU transcription factor Oct4. Cell 1998; 95:379-91.

[13] Niwa H. Molecular mechanism to maintain stem cell renewal of ES cells. Cell Str \& Func 2001; 26:13748.

[14] Niwa H, J Miyazaki, A Smith. Quantitative expression of Oct-3/4 defines differentiation, dedifferentiation or self-renewal of ES cells. Nat Genet 2000; 24:372-6.

[15] Klemm JD, MA Rould, R Aurora, W Herr, CO Pabo. Crystal structure of the Oct-1 POU domain bound to an octamer site: DNA recognition with tethered DNA-binding modules. Cell 1994; 77:21-32.

[16] Brehm A, K Ohbo, H Scholer. The carboxy-terminal transactivation domain of Oct4 acquires cell specificity through the POU domain. Mol Cell Biol 1997; 17:15462.

[17] Vigano MA, LM Staudt. Transcriptional activation by Oct-3: evidence for a specific role of the POU-specific domain in mediating functional interaction with Oct-1. Nucleic Acids Res 1996; 24:2112-8.

[18] Brehm A, C Ovitt, H Scholer. Oct4: more than just a POUerful marker of the mammalian germline? Apmis 1998; 106:114-24.

[19] Brehm A, K Ohbo, W Zwerschke, V Botquin, P JansenDurr, H Scholer. Synergism with germ line transcription factor Oct4: viral oncoproteins share the ability to mimic a stem cell-specific activity. Mol Cell Biol 1999; 19: 263543.

[20] Pesce M, X Wang, D Wolgemuth, H Scholer. Differential expression of the Oct4 transcription factor during mouse germ cell differentiation. Mech Dev 1998; 71:89-98.

[21] Palmieri S, W Peter, H Hess, H Scholer. Oct4 transcription factor is differentially expressed in the mouse embryo during establishment of the first two extraembryonic cell lineages involved in implantation. Dev Biol 1994; 166:259-67.

[22] Hansis C, J Grifo, L Krey. Oct4 expression in inner cell mass and trophectoderm of human blastocysts. Mol Hum Reprod 2000; 6:999-1004.

[23] Hansis C, Y Tang, J Grifo, L Krey. Analysis of Oct4 expression and ploidy in individual human blastomeres. Mol Hum Reprod 2001; 7:155-61.

[24] Ben-Shushan E, E Pikarsky, A Klar, Y Bergman. Extinction of Oct-3/4 gene expression in embryonal carcinoma $\mathrm{x}$ fibroblast somatic cell hybrids is accompanied by changes in the methylation status, chromatin structure, and transcriptional activity of the Oct-3/4 upstream region. Mol Cell Biol 1993; 13:891-901.

[25] Yeom Y, G Fuhrmann, C Ovitt, A Brehm, K Ohbo, M Gross, K Hubner, H Scholer. Germline regulatory element of Oct4 specific for the totipotent cycle of embryonal cells. Integrated Ann Indexes 1996; 122:881-94.

[26] Nordhoff V, K Hubner, A Bauer, I Orlova, A Malapetsa, $\mathrm{H}$ Scholer. Comparative analysis of human, bovine, and murine Oct4 upstream promoter sequences. Mamm Genome 2001; 12:309-17.
[27] Hummelke GC, AJ Cooney. Germ cell nuclear factor is a transcriptional repressor essential for embryonic development. Front Biosci 2001; 6:D1186-91.

[28] Fuhrmann G, AC Chung, KJ Jackson, G Hummelke, A Baniahmad, J Sutter, I Sylvester, HR Scholer, AJ Cooney. Mouse germline restriction of Oct4 expression by germ cell nuclear factor. Dev Cell 2001; 1:377-87.

[29] Jaenisch R. DNA methylation and imprinting: why bother? Trends Genet 1997; 13:323-9.

[30] Goto T, J Adjaye, C Rodeck, M Monk. Identification of genes expressed in human primordial germ cells at the time of entry of the female germ line into meiosis. Mol Hum Reprod 1999; 5:851-60.

[31] Pesce M, M Gross, H Scholer. In line with our ancestors: Oct4 and the mammalian germ. Bioessays 1998; 20:722-32.

[32] Liu L, R Roberts. Silencing of the gene for the beta subunit of human chorionic gonadotropin by the embryonic transcription factor Oct-3/4. J Biol Chem 1996; 271: 16683-9.

[33] Liu L, D Leaman, M Villalta, R Roberts. Silencing of the gene for the alpha-subunit of human chorionic gonadotropin by the embryonic transcription factor Oct-3/4. Mol Endocrinol 1997; 11:1651-8.

[34] Hromas R, R Costa. The hepatocyte nuclear factor-3/ forkhead transcription regulatory family in development, inflammation, and neoplasia. Crit Rev Oncol Hematol 1995; 20:12940.

[35] Guo Y, R Costa, H Ramsey, T Starnes, G Vance, K Robertson, M Kelley, R Reinbold, H Scholer, R Hromas. The embryonic stem cell transcription factors Oct4 and FoxD3 interact to regulate endodermal-specific promoter expression. Proc Natl Acad Sci USA 2002; 99:3663-7.

[36] Yamamoto H, ML Flannery, S Kupriyanov, J Pearce, SR McKercher, GW Henkel, RA Maki, Z Werb, RG Oshima. Defective trophoblast function in mice with a targeted mutation of Ets2. Genes Dev 1998; 12:1315-26.

[37] Ezashi T, D Ghosh, RM Roberts. Repression of Ets-2induced transactivation of the tau interferon promoter by Oct4. Mol Cell Biol 2001; 21:7883-91.

[38] Butteroni C, FM De, H Scholer, M Pesce. Phage display screening reveals an association between germline-specific transcription factor Oct4 and multiple cellular proteins. J Mol Biol 2000; 304:52940.

[39] Scholer H, T Ciesiolka, P Gruss. A nexus between Oct4 and E1A: implications for gene regulation in embryonic stem cells. Cell 1991; 66:291-304.

[40] Curatola AM, C Basilico. Expression of the K-fgf protooncogene is controlled by 3 ' regulatory elements which are specific for embryonal carcinoma cells. Mol Cell Biol 1990; 10:2475-84.

[41] Yuan H, N Corbi, C Basilico, L Dailey. Developmentalspecific activity of the FGF4 enhancer requires the synergistic action of Sox-2 and Oct-3. Genes Dev 1995; 9: 263545.

[42] Ambrosetti DC, C Basilico, L Dailey. Synergistic activation of the fibroblast growth factor 4 enhancer by Sox 2 and Oct-3 depends on protein-protein interactions facilitated by a specific spatial arrangement of factor binding sites. Mol Cell Biol 1997; 17:6321-9. 
[43] Ambrosetti DC, HR Scholer, L Dailey, C Basilico. Modulation of the activity of multiple transcriptional activation domains by the DNA binding domains mediates the synergistic action of Sox-2 and Oct-3 on the fibroblast growth factor4 enhancer. J Biol Chem 2000; 275:23387-97.

[44] Tomioka M, M Nishimoto, S Miyagi, T Katayanagi, N Fukui, H Niwa, M Muramatsu, A Okuda. Identification of Sox-2 regulatory region which is under the control of Oct3/4-Sox-2 complex. Nucleic Acids Res 2002; 30:3202-13.

[45] Nishimoto M, A Fukushima, A Okuda, M Muramatsu. The gene for the embryonic stem cell coactivator UTF1 carries a regulatory element which selectively interacts with a complex composed of Oct-3/4 and Sox-2. Mol Cell Biol 1999; 19:5453-65.

[46] Wakayama T, R Yanagimachi. Cloning the laboratory mouse. Semin Cell Dev Biol 1999; 10:253-8.

[47] Wakayama T, H Tateno, P Mombaerts, R Yanagimachi. Nuclear transfer into mouse zygotes. Nat Genet 2000; 24:108-9.
[48] Wakayama T, R Yanagimachi. Mouse cloning with nucleus donor cells of different age and type. Mol Reprod Dev 2001; 58:376-83.

[49] Du Z, H Cong, Z Yao. Identification of putative downstream genes of Oct4 by suppression-subtractive hybridization. Biochem Biophys Res Comm 2001; 282: 701-6.

[50] Boiani M, S Eckardt, H Scholer, K McLaughlin. Oct4 distribution and level in mouse clones: consequences for pluripotency. Genes Dev 2002; 16:1209-19.

[51] Palumbo C, RK van, A Gillis, GR van, MH de, J Oosterhuis, ZE van, L. Looijenga. Expression of the PDGF alphareceptor $1.5 \mathrm{~kb}$ transcript, OCT4, and c-Kit in human normal and malignant tissues. Implications for the early diagnosis of testicular germ cell tumours and for our understanding of regulatory mechanisms. J Path 2002; 196:467-77.

[52] Monk M, C Holding. Human embryonic genes re-expressed in cancer cells. Oncogene 2001; 20:8085-91.

[53] Jin T, DR Branch, X Zhang, S Qi, B Youngson, PE Goss. Examination of POU homeobox gene expression in human breast cancer cells. Int J Cancer 1999; 81:104-12. 